\title{
Liver metabolic perturbations of heat-stressed lactating dairy cows
}

\author{
Caiyun Fan ${ }^{1, a}$, Di Su' ${ }^{1, a}$, He Tian' ${ }^{2}$ Xiaojiao Li', Yu Li', Lei Ran', Ruiting Hu', and Jianbo Cheng ${ }^{1}$ **
}

\author{
* Corresponding Author: Jianbo Cheng \\ Tel: +86-551-65786328, Fax: +86-551-65786328, \\ E-mail: chengjianboahau@163.com \\ ${ }^{1}$ College of Animal Science and Technology, Anhui \\ Agricultural University, Hefei 230036, China \\ 2 Institute of Genetics and Developmental Biology, \\ Chinese Academy of Sciences, Beijing 100101, China \\ ${ }^{a}$ These authors contributed equally. \\ ORCID \\ He Tian \\ https://orcid.org/0000-0002-5388-0297 \\ Jianbo Cheng \\ https://orcid.org/0000-0003-0141-0514
}

Submitted Aug 4, 2017; Revised Oct 1, 2017; Accepted Feb 14, 2018
Objective: The objective of the present study was to elucidate the mechanism underlying liver metabolic perturbations in dairy cows exposed to heat stress (HS).

Methods: Liquid chromatography massabl spectrometry was used to analyze metabolic differences in livers of 20 dairy cows, with and without exposure to HS.

Results: The results revealed 33 potential metabolite candidate biomarkers for the detection of HS in dairy cows. Fifteen of these metabolites (glucose, lactate, pyruvate, acetoacetate, $\beta$-hydroxybutyrate, fumaric acid, citric acid, choline, glycine, proline, isoleucine, leucine, urea, creatinine, and orotic acid) were previously found to be potential biomarkers of HS in plasma or milk, discriminating dairy cows with and without HS.

Conclusion: All the potential diagnostic biomarkers were involved in glycolysis, amino acid, ketone, tricarboxylic acid, or nucleotide metabolism, indicating that HS mainly affected energy and nucleotide metabolism in lactating dairy cows.

Keywords: Metabolomics; Liver; Heat Stress; Dairy Cows

\section{INTRODUCTION}

Heat stress (HS) negatively impacts the performance of dairy cows, for example, reducing milk yields and quality, damaging animal health, and causing reproductive disorders [1-7]. Major economic losses due to HS in dairy cows are inevitable in most parts of the world, including southern China, the U.S., and Germany [8-10]. Considering ongoing rises in temperatures worldwide and the adoption of modern molecular genetic technologies aimed at increasing milk productivity, better responses to the problem of HS faced by the dairy industry need to be devised [2].

In mammals, cell metabolism responds to HS via self-regulatory mechanisms $[1,11]$. These mechanisms maintain body homeostasis in response to a rise in the temperature and humidity index (THI), which is used to measure the HS status of dairy cows [5,12]. Physiological changes in response to HS are complicated and multifactorial. Therefore, studies of HS mechanisms in dairy cows have focused on whole body-related factors, such as dry matter intake, milk yield, rectal temperature, respiration rate, and heart rate, as well as molecular changes involving genes, proteins, and metabolites [1,3-5]. Our previous metabolomic research on blood and milk in dairy cows affected by HS revealed metabolic perturbations involving carbohydrates, amino acids, and lipids $[4,5]$. However, the mechanisms of HSinduced disturbances in dairy cows remain far from clear, as cellular metabolism in multiple organs responds to HS and other exogenous stimuli. Systematic whole-body metabolic responses of dairy cows to HS need to be studied to elucidate the mechanisms of HS in dairy cows and identify robust diagnostic biomarkers that can facilitate the selection of dairy cows with good tolerance to a high THI.

The liver is one of the major organs involved in the metabolism of proteins, lipids, glu- 
cose, amino acids, vitamins, and hormones [13,14], all of which have been shown to be disturbed in dairy cows exposed to HS $[4,5]$. Therefore, studies of liver cellular metabolism should provide major insights into HS mechanisms in dairy cows.

Untargeted metabolomics using liquid chromatography mass spectrometry (LC-MS) have enabled the detection of tens of thousands of metabolic features of cells, biofluids, and tissues; hence, LC-MS has become the primary platform for metabolic profiling experiments [15]. In our previous studies, using LC-MS, we discovered several potentially valuable biomarkers in blood and milk for diagnosing HS in dairy cows $[4,5]$. In this study, we investigated metabolic differences in the livers of cows with and without HS using LC-MS to explore perturbations of metabolic pathways. In an attempt to provide a systematic perspective on the physiological mechanisms of HS in greater depth, we discuss the findings of the present study in conjunction with those of our earlier discoveries in blood and milk of heat-stressed dairy cows.

\section{MATERIALS AND METHODS}

\section{Reagents and standards}

High-performance liquid chromatography (HPLC) grade acetonitrile, isopropanol, methyl tert-butyl ether, water, formic acid, and ammonium formate were purchased from Merck (Darmstadt, Germany). All standard compounds were purchased from Sigma-Aldrich (St. Louis, MO, USA). All internal standard compounds were purchased from Cambridge Isotope Laboratories, Inc. (Tewksbury, MA, USA), including $\left[2,3,3,4,4-\mathrm{D}_{5}\right]$-L-glutamate, $\left[2,3,3-\mathrm{D}_{3}\right]$-L-aspartate, $\left[{ }^{13} \mathrm{C}_{4}\right]-\mathrm{L}$ asparagine, $\left[2,3,3-\mathrm{D}_{3}\right]$-L-serine, $\left[2,3,3,4,4-\mathrm{D}_{5}\right]$-L-glutamine, [Ring-2,4- $\mathrm{D}_{2}$; alpha, beta, beta- $\left.\mathrm{D}_{3}\right]$-L-histidine, $\left[\mathrm{D}_{5}\right]$-L-threonine, $\left[\mathrm{D}_{7}\right]$-L-alanine, $\left[\mathrm{D}_{7}\right]$-L-arginine, $\left[\mathrm{D}_{7}\right]$-L-proline, $\left[\mathrm{D}_{8}\right]$ L-valine, $\left[\mathrm{D}_{3}\right]$-L-methionine, $\left[\mathrm{D}_{10}\right]$-L-isoleucine, $\left[\mathrm{D}_{10}\right]$-L-leucine, $\left[\mathrm{D}_{8}\right]$-L-phenylalanine, and $\left[\mathrm{D}_{8}\right]$-L-tryptophan, $\left[\mathrm{D}_{9}\right]$-L-lysine.

\section{Experimental groups}

All experiments involving animals were conducted according to the principles of the Anhui Agricultural University Animal Care and Use Committee (Hefei, China). The study consisted of 20 Holstein cows, which were raised in a closed type cowshed to minimize the effects of photoperiods on their metabolism between different seasons. Ten cows were assigned to a HS group, and another 10 were assigned to a non-HS group. All the animals were fed the same diet. Data on the numbers, lactation days, 305-day milk yield, and body weight of the dairy cows are presented in Table 1. Details on feed composition, temperature, humidity, rectal temperature, respiration rate, and production characteristics of the two groups of dairy cows are provided in Supplementary Tables S1-S4 in the supplementary material. Rectal temperature and respiration rate,
Table 1. Characteristics of the Holstein dairy cows used in the study

\begin{tabular}{lcc}
\hline Item & HS & Non-HS \\
\hline Lactation days & $142.1 \pm 27.2$ & $137.6 \pm 23.6$ \\
Parity & $4.4 \pm 1.2$ & $4.2 \pm 1.3$ \\
305 days milk yield $(\mathrm{kg} / 305 \mathrm{~d})$ & $8,911.2 \pm 651.4$ & $8,973.7 \pm 727.5$ \\
No. of cows & 10 & 10 \\
Average weight $(\mathrm{kg})$ & $709.2 \pm 54.7$ & $700.6 \pm 48.5$ \\
\hline HS, heat stress. & &
\end{tabular}

dried matter intake, milk yields, protein, milk urea nitrogen, and somatic cell count were significantly different $(\mathrm{p}<0.01)$ between non-HS and HS groups.

\section{Sample collection}

Liver samples were collected immediately after the dairy cows ( $n=10$ in each group) had been slaughtered. The samples were obtained from the HS group in August (summer season) after the natural THI had increased from 72.3 to 86.7 over a 1 -month period and had remained stable at 81 for 1 week. Samples were obtained from the HS-free group $(n=10)$ in November (fall season) after the THI had gradually decreased from 60 to 47 over a 1-month period. The THI was calculated using the following formula, according to criteria developed by the NRC in 1971: THI $=(1.8 \times \mathrm{Tdb}+32)-[(0.55-0.0055 \times \mathrm{RH})$ $\times(1.8 \times \mathrm{Tdb}-26.8)]$, where Tdb was the dry-bulb temperature $\left({ }^{\circ} \mathrm{C}\right)$ and $\mathrm{RH}$ was the relative humidity (\%). The liver samples (approximately $1 \mathrm{~g}$ ) were clipped and washed quickly 10 times in phosphate-buffered saline ( $\mathrm{pH}$ 7.2 to $7.4: \mathrm{KCl} 2.7 \mathrm{mM}, \mathrm{NaCl}$ $137 \mathrm{mM}, \mathrm{KH}_{2} \mathrm{PO}_{4} 2 \mathrm{mM}$, and $\mathrm{Na}_{2} \mathrm{HPO}_{4} 10 \mathrm{mM}$ ). A portion of the tissue samples was immediately stored in liquid nitrogen.

\section{Sample preparation}

The samples were prepared with reference to the previous literature but with some modifications [16]. Briefly, beads were added to $30 \mathrm{mg}$ of a liver sample using a metal spatula, which was precleaned with HPLC-grade methanol. Tissues were homogenized in a bead ruptor by quickly spinning the samples at $4^{\circ} \mathrm{C}$ at a speed of $4 \mathrm{~m} / \mathrm{s}$ for $5 \mathrm{~s}$ and two cycles. The samples were then centrifuged at $12,000 \mathrm{rpm}$ at $4^{\circ} \mathrm{C}$ for $5 \mathrm{~min}$. The supernatants were collected and then dried in a micVac vacuum centrifugal concentrator. Finally, the dried samples were reconstituted in $1 \mathrm{~mL}$ of initial mobile phase for LC-MS analysis.

\section{LC-MS/MS analysis}

The conditions of chromatography were as described previously [4]. An Ultimate 3000 UHPLC system was coupled to an Q-Exactive MS instrument (Thermo Scientific, Bremen, Germany). Chromatographic separation was conducted using a Waters HSS $\mathrm{T}_{3}$ column $(1.8 \mu \mathrm{m}, 100 \mathrm{~mm} \times 2.1 \mathrm{~mm})$. The Q-Exactive MS operation was carried out in both positive and 
negative ion modes. The resolution of the full scan was set at 35,000 , and the scan range was $m / z 50$ to 400 .

To generate the tandem mass spectrometry data, the resolution was set at 17,500: automatic gain control target, $1 \mathrm{e}^{6}$; maximum ion injection time, $100 \mathrm{~ms}$; isolation window, $1 \mathrm{~m} / \mathrm{z}$; and stepped normalized collision energy at 25,35, and 45 .

High-resolution MS, isotope abundance ratios, MS/MS, the Human Metabolome database, the METLIN database, a literature search, and standard comparisons were employed to identify ion structures.

\section{Data handling}

Raw data were converted to mzXML format using MassMatrix MS Data File Conversion Tools (www.massmatrix.net). Full details of the next step in the data analysis have been described in our previous paper [4]. Briefly, the files were processed using an open-source XCMS package (version 1.20.1) in R statistical software (version 2.10.0) for peak discrimination, filtering, and alignment. The resulting two-dimensional matrices, including observations (sample names) in columns, variables ( $\mathrm{m} / \mathrm{z}$-retention time pairs) in rows, and peak areas, were imported into the SIMCA-P 13.0 software package (Umetrics AB, Umeå, Sweden) for multivariate analysis and discovery of metabolite candidates.

The sensitivity and specificity of all candidate biomarkers were evaluated by plotting receiver operating characteristic (ROC) curves using SPSS (version 13.0), and calculating the area under the curves (AUC). The discriminatory power of candidate biomarkers was ranked and visualized by heat maps.

\section{RESULTS}

Quality assessment of the LC-MS data

A multivariate analysis of quality control samples showed that the stability of the analytical system was acceptable [4] and that the deviation in peak areas was less than two standard deviations. Representative total ion chromatograms of the liver samples are shown in Supplementary Figure S1 in the supporting data.

\section{Comparison of the metabolic profiles of HS and non- HS groups}

The orthogonal partial least squares discriminant analysis (OPLS-DA) plots for analyzing the LC-MS data of the dairy cows showed a clear separation between the non-HS and HS groups, without any overlap (Figure 1A). This model produced one predictive component and two orthogonal components, with satisfactory modeling and predictive abilities: $\left(R^{2}(\mathrm{X})=\right.$ $56.9 \%, R^{2}(\mathrm{Y})=86.5 \%$, and $\left.Q^{2}(\mathrm{cum})=64.9 \%\right)$. To avoid model overfitting, seven rounds of cross-validation across three components was performed. Validation with 999 random permutation tests produced intercepts $R^{2}=0.291$ and $Q^{2}=$ -0.381 for all the data (Figure 1B). Overall, the results indicated that the predictive capabilities of the OPLS-DA model of the LC-MS data were reliable.

\section{Identification of metabolic candidates and their structures}

The LC-MS data analysis showed that 321 metabolites differed in the non-HS versus the HS groups, with 39 of these metabolites remaining after elimination of redundant variables using a combination of extracted ion chromatograms (XICs) and the R-package CAMERA. Finally, 33 candidates were identified. Of these, 21 and 12 metabolic candidate biomarkers were confirmed in positive and negative ion modes, respectively.

\section{ROC evaluation of potential diagnostic biomarkers}

The diagnostic power of the 33 metabolic candidates to indi-
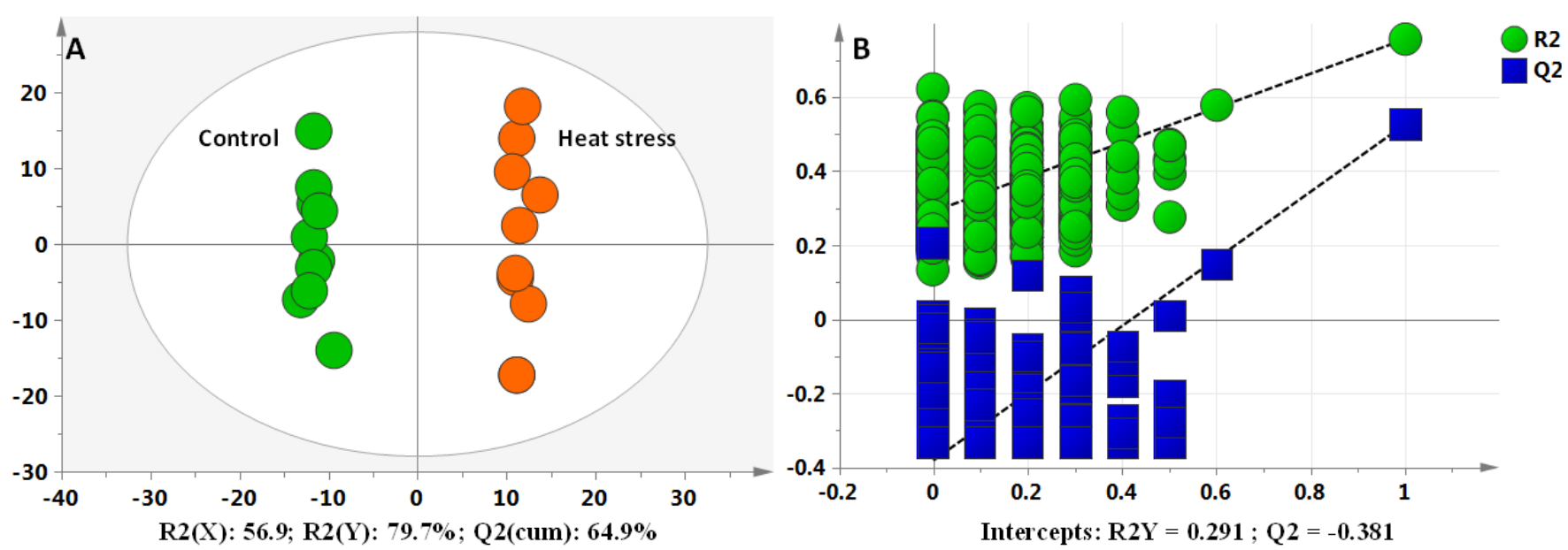

Figure 1. Differentiation of control and heat stress (HS) groups using multivariate analysis. The orthogonal partial least squares discriminant analysis (OPLS-DA) plots of liquid chromatography mass spectrometry (LC-MS) data for the plasma metabolomes (A). Validation plots of the partial least squares discriminant analysis models acquired through 999 permutation tests for LC-MS data of liver metabolome (B). 
cate HS status was assessed by ROC analyses (Figure 2). The discriminatory power of each candidate was ranked using heat maps, and only those with an AUC $>0.65$ were accepted. Eight candidates (glucose, lactate, $\beta$-hydroxybutyrate [BHBA], fumaric acid, glycine, uric acid, uridine, and creatinine) were identified as potentially reliable diagnostic biomarkers of the HS status of lactating dairy cows based on an AUC $>0.85$, which is considered to denote a highly sensitive and specific diagnostic biomarker [4].

\section{Metabolic alterations}

The concentrations of the candidate metabolites are listed in Table 2. HS-induced alterations in glycolysis-related metabolites were observed in glucose, lactate, pyruvate, fructose 1,6-bisphosphate, D-glyceraldehyde 3-phosphate, and glucose 6-phosphate/fructose 6-phosphate. The concentrations of the first three metabolites increased 1.54- to 1.89-fold in the HS group compared with the non-HS group $(\mathrm{p}<0.01)$, whereas those of the last three metabolites decreased 0.44- to 0.78 -fold $(\mathrm{p}<0.01)$. The concentrations of acetoacetate and BHBA (ketone metabolites) increased 1.52- and 1.98-fold, respectively, in the HS group compared with the non-HS group $(\mathrm{p}<0.001)$. The concentrations of tricarboxylic acid (TCA)-related metabolites, such as malic acid, ketoglutaric acid, succinic acid, fumaric acid, citric acid, and oxalacetic acid, were significantly changed in the HS group compared with the non-HS group, with concentrations of the first four metabolites decreased 0.50 - to 0.62 -fold $(\mathrm{p}<0.01)$ and those of the last two metabolites increased 1.37 - and 1.78 -fold $(\mathrm{p}<0.05)$, respectively. The concentration of choline increased 1.59-fold in the HS group compared with the non-HS group $(\mathrm{p}<0.01)$. Metabolites, including glycine, asparagine, glutamate, threonine, proline, valine, methionine, isoleucine, and histidine, involved in amino acid metabolism were down-regulated 0.63 - to 0.78 -fold in the HS group as compared with the non-HS group ( $\mathrm{p}<0.01)$, with the exception of leucine, urea, and creatinine, which were upregulated 1.24- to 1.70 -fold ( $\mathrm{p}<0.01)$. HS-induced alterations in nucleotide-related metabolites (orotic acid, uridine $5^{\prime}$-monophosphate, uric acid, adenosine monophosphate, uridine, and uracil) were also observed, with the concentrations increased 1.46- to 2.26-fold in the HS group as compared with the non-HS group $(\mathrm{p}<0.01)$.

\section{DISCUSSION}

\section{Potential diagnostic biomarkers of HS status}

In this study, 33 metabolite biomarkers were identified that may have potential in detecting the HS status of dairy cows. As can be seen, 15 (glucose, lactate, pyruvate, acetoacetate, BHBA, fumaric acid, citric acid, choline, glycine, proline, isoleucine, leucine, urea, creatinine, and orotic acid) of the 33 potential biomarkers were also discovered in blood or milk in our previous studies $[4,5]$. Thus, these 15 candidate biomarkers best represent the global HS metabolic status of dairy cows. These 15 metabolites may be used to provide accurate information on the degree of HS in dairy cows. They may also facilitate the selection of breeds and individual animals that can best tolerate a high THI.
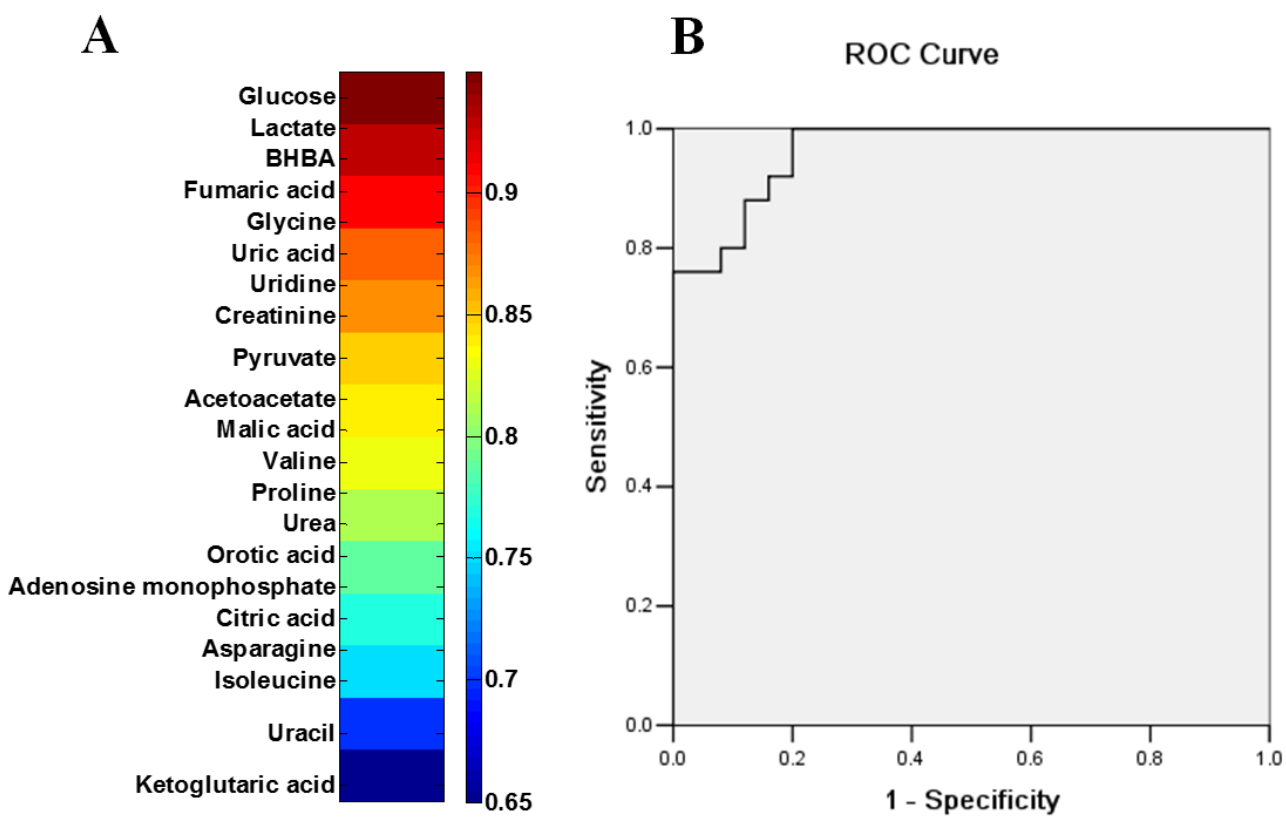

Figure 2. Visualization of the discriminatory powers of individual and combined diagnostic biomarkers. Heat maps showing the discriminative capacities of selected biomarkers (A). The red and blue colors represent high and low areas under the curve (AUC), respectively. Receiver operating characteristic (ROC) curve of the combined potential biomarkers (AUC $>0.85)(B)$. 
Table 2. Candidate metabolites of HS groups identified by LC-MS/MS

\begin{tabular}{|c|c|c|c|c|c|c|}
\hline No. & Metabolic pathway & Metabolite & Identification $\mathrm{m} / \mathrm{z}^{1)}$ & Retention time & p-value & $\mathrm{FC}^{2)}$ \\
\hline 1 & Glycolysis & Glucose $^{3)}$ & 203.05202 & 1.43 & $7.65 \times 10^{-4}$ & 1.54 \\
\hline 2 & Glycolysis & Lactate $^{3)}$ & 89.02436 & 2.57 & $1.16 \times 10^{-5}$ & 1.89 \\
\hline 3 & Glycolysis & Pyruvate $^{3)}$ & 87.00854 & 3.59 & $6.49 \times 10^{-3}$ & 1.63 \\
\hline 4 & Glycolysis & Fructose 1,6-bisphosphate ${ }^{4)}$ & 338,98867 & 1.32 & $3.63 \times 10^{-3}$ & 0.57 \\
\hline 5 & Glycolysis & D-Glyceraldehyde 3-phosphate & 175.1191 & 0.95 & $2.26 \times 10^{-3}$ & 0.78 \\
\hline 6 & Glycolysis & Glucose 6-phosphate/Fructose 6-phosphate ${ }^{3)}$ & 259.02419 & 1.33 & $6.19 \times 10^{-5}$ & 0.44 \\
\hline 7 & Ketone & Acetoacetate $e^{3)}$ & 101.02421 & 1.41 & $3.01 \times 10^{-4}$ & 1.52 \\
\hline 8 & Ketone & $\beta$-hydroxybutyrate & 103.04001 & 4.75 & $1.21 \times 10^{-4}$ & 1.98 \\
\hline 9 & TCA & Malic acid ${ }^{3)}$ & 133.01427 & 2.03 & $1.85 \times 10^{-3}$ & 0.50 \\
\hline 10 & TCA & Ketoglutaric acid ${ }^{4)}$ & 145.01411 & 2.41 & $5.33 \times 10^{-4}$ & 0.58 \\
\hline 11 & TCA & Succinic acid ${ }^{3)}$ & 117.01915 & 4.53 & $3.61 \times 10^{-4}$ & 0.62 \\
\hline 12 & TCA & Fumaric acid ${ }^{3)}$ & 115.00351 & 2.03 & $7.52 \times 10^{-3}$ & 0.61 \\
\hline 13 & TCA & (itric acid ${ }^{3)}$ & 191.01925 & 3.40 & $2.63 \times 10^{-3}$ & 1.78 \\
\hline 14 & TCA & Oxalacetic acid ${ }^{3)}$ & 130.99841 & 1.74 & $1.55 \times 10^{-2}$ & 1.37 \\
\hline 15 & Lipid & Choline $^{3)}$ & 104.10675 & 1.39 & $1.35 \times 10^{-3}$ & 1.59 \\
\hline 16 & Amino acid & Glycine $^{3)}$ & 76.039179 & 1.30 & $5.63 \times 10^{-3}$ & 0.78 \\
\hline 17 & Amino acid & Asparagine $^{3)}$ & 133.06061 & 1.32 & $4.61 \times 10^{-4}$ & 0.63 \\
\hline 18 & Amino acid & Glutamate $^{3)}$ & 148.06034 & 1.42 & $7.42 \times 10^{-4}$ & 0.63 \\
\hline 19 & Amino acid & Threonine $e^{3)}$ & 120.06552 & 1.41 & $1.94 \times 10^{-3}$ & 0.67 \\
\hline 20 & Amino acid & Proline $e^{3)}$ & 116.07047 & 1.64 & $1.98 \times 10^{-3}$ & 0.71 \\
\hline 21 & Amino acid & Valine $^{3)}$ & 118.08601 & 2.35 & $5.75 \times 10^{-3}$ & 0.76 \\
\hline 22 & Amino acid & Methionine $e^{3)}$ & 150.05811 & 2.87 & $2.49 \times 10^{-3}$ & 0.66 \\
\hline 23 & Amino acid & Isoleucine ${ }^{3)}$ & 132.10178 & 4.76 & $1.76 \times 10^{-3}$ & 0.72 \\
\hline 24 & Amino acid & Histidine $^{3)}$ & 156.07653 & 1.29 & $2.39 \times 10^{-3}$ & 0.75 \\
\hline 25 & Amino acid & Leucine $^{3)}$ & 132.10181 & 4.84 & $8.71 \times 10^{-3}$ & 1.24 \\
\hline 26 & Amino acid & Urea $^{4)}$ & 121.07191 & 1.45 & $3.59 \times 10^{-3}$ & 1.66 \\
\hline 27 & Amino acid & Creatinine $^{4)}$ & 114.06602 & 1.58 & $2.29 \times 10^{-3}$ & 1.70 \\
\hline 28 & Nucleotide & Orotic acid ${ }^{4)}$ & 155.00783 & 2.20 & $2.56 \times 10^{-3}$ & 1.51 \\
\hline 29 & Nucleotide & Uridine $^{4)} 5^{\prime}$-monophosphate ${ }^{4)}$ & 323.02859 & 2.26 & $4.27 \times 10^{-4}$ & 2.26 \\
\hline 30 & Nucleotide & Uric acid ${ }^{4)}$ & 167.02098 & 3.42 & $2.56 \times 10^{-3}$ & 1.64 \\
\hline 31 & Nucleotide & Adenosine $^{4)}$ monophosphate ${ }^{4)}$ & 346.0558701 & 2.94 & $7.93 \times 10^{-3}$ & 1.73 \\
\hline 32 & Nucleotide & Uridine $^{4)}$ & 245.07643 & 4.78 & $8.44 \times 10^{-3}$ & 1.46 \\
\hline 33 & Nucleotide & Uracil $^{4)}$ & 113.03432 & 4.78 & $8.00 \times 10^{-3}$ & 1.49 \\
\hline
\end{tabular}

HS, heat stress; LC-MS, liquid chromatography mass spectrometry.

1) The non-italicized and italicized $\mathrm{m} / \mathrm{z}$ values are metabolites detected separately in positive and negative ion modes.

${ }^{2)}$ Fold change of metabolite concentration (HS/non-HS).

3) Metabolites verified by standard compounds.

4) Metabolites putatively identified by database comparison and characteristic fragmentation.

$p$-value, independent $t$-test for non-HS vs HS.

\section{Disturbed glycolysis}

In the present study, HS-induced upregulation of lactate and pyruvate in the liver $(\mathrm{p}<0.01)$ pointed to increased anaerobic glycolysis. In common with this finding, lactate and pyruvate were also upregulated in blood and milk in heat-stressed dairy cows in our previous studies $[4,5]$. This finding indicates that there are characteristic increases in anaerobic metabolism in heat-stressed dairy cows. The role of HS in reducing dry matter intake of dairy cows, which leads to a negative energy balance (NEB), is well known [3,11]. Our previous findings suggested that HS resulted in increases in oxygen consumption by myocardia and muscles and decreases in oxygen supply, leading to upregulation of anaerobic glycolysis and anaerobic cell respiration [4]. In comparison to high-yielding ATP following complete oxidation of a single molecule of glucose, low-yielding ATP via anaerobic glycolysis further aggravates NEB [17]. The vast majority of whole-body gluconeogenesis is realized in the liver [18]. Thus, the increased glucose concentrations found in the liver in the present study are the result of activated gluconeogenesis metabolism as a result of a NEB. The aforementioned is an adaptive mechanism for maintaining energy homeostasis in response to HS. In our previous study, we found a reduced level of glucose in plasma of heat-stressed dairy cows [4]. We hypothesize that HS induced a reduction in intake and that this contributed to a shortage of glucose supply in the whole body. In muscle and other tissues, increased 
glycolysis could be an alternative route to produce ATP when mitochondrial function is compromised or oxygen levels are low, thereby further reducing the level of glucose in the circulatory system. Upregulation of gluconeogenesis metabolism in the liver is not sufficient to compensate for this glucose shortage. Thus, the alterations in glucose metabolism observed in liver in the present study differed from those observed in plasma. A previous study reported down-regulation of fructose 1,6-bisphosphate, D-glyceraldehyde 3-phosphate, glucose 6-phosphate, and fructose 6-phosphate, pointing to HS-induced disturbances in glycolysis [19]. Seven metabolites (lactate, pyruvate, $\beta$-BHBA, glycine, proline, isoleucine, and urea) detected in our previous study of plasma and milk in dairy cows exposed to HS were also perturbed in the liver samples in the present study (Figure 3). This finding points to a whole body response, which includes glycolysis, fat metabolism, and energy redistribution, of dairy cows to HS.

\section{Ketone metabolism}

In the present study, both acetoacetate and $\beta$-BHBA, metabolites of acetyl-CoA, were upregulated in the HS group. Under NEB states, $\beta$-oxidation of free fatty acids is increased to produce energy to fulfil the demands of the body [20]. Accordingly, in the present study, the concentration of acetylCoA was raised, and ketone metabolites were increased in the HS group. These perturbations were in line with the results found in plasma and milk of heat-stressed dairy cows in our previous studies $[4,5]$.

\section{Tricarboxylic acid intermediates}

The present study revealed marked changes in the concen-

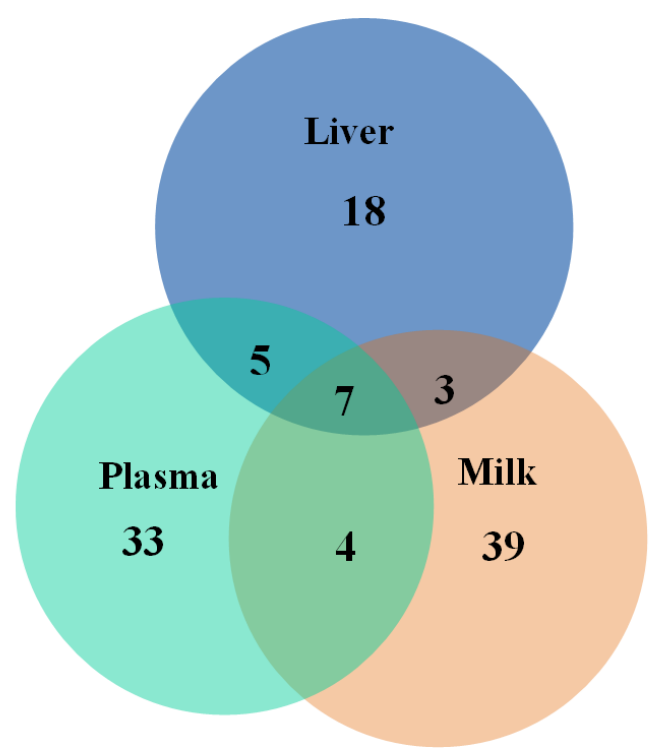

Figure 3. Venn diagram showing the numbers of unique and common potential biomarkers identified by the metabolomics studies on liver, plasma, and milk, respectively. trations of TCA in the HS group, indicating mitochondrial dysfunction and an energy crisis [19]. These changes included increased concentrations of citric acid and oxalacetic acid, as well as decreased concentrations of malic acid, ketoglutaric acid, succinic acid, and fumaric acid. This finding suggested that HS exerted different effects on the catalysis of enzymes in different steps of the biochemical reactions with TCA. HSinduced upregulation of $\beta$-BHBA provided further evidence of perturbation of mitochondrial function.

\section{Amino acid metabolism}

The liver is the major site of gluconeogenesis [21]. In the present study, amino acids, including glycine, asparagine, glutamate, threonine, proline, valine, methionine, isoleucine, and histidine, were down-regulated in the HS group, pointing to increased conversion of these metabolites into glucose to maintain homeostasis and supply energy during periods of NEB in dairy cows [22]. Mobilization of body fat may lead to the formation of ketone bodies, such as acetoacetate, acetone, and BHBA, which can then be used for energy supply or milk fat synthesis $[4,5]$.

The finding of elevated levels of creatinine and urea in the present study was in accordance with that found in plasma of heat-stressed dairy cows in our previous studies $[4,5]$. The time points could point to the use of skeletal muscle energy reserves and mobilization of muscle tissues during periods of $\mathrm{NEB}$ in dairy cows to fulfill energy demands [4,5]. Leucine, which is not the same as glycogenic amino acids, was increased in dairy cows exposed to HS, likely due to an increased supply from HS plasma.

\section{Nucleotide metabolism}

Perturbations of nucleotide metabolites, such as orotic acid, uridine 5'-monophosphate, uric acid, adenosine monophosphate, uridine, and uracil, were observed in the present study in the HS groups.

Adenosine monophosphate, a compound in DNA and RNA, is involved in purine-pyrimidine metabolism. HS-induced increases of these metabolites suggested that nucleotide metabolism was altered by HS [23]. Previous studies revealed correlations between genome-wide associations and heat tolerance in dairy cattle $[24,25]$. This finding suggests that nucleotide metabolites are potential biomarkers in selection for tolerance to HS in dairy cattle.

We can get a picture of how HS affects metabolite profile by putting all the research results of plasma, milk, and live of HS dairy cows together. First in the liver, glycolysis was increased in HS groups, with the sign of up-regulation of pyruvate and lactate. Although the different alterations were identified for intermediates from glucose to pyruvate, such as fructose 1,6-bisphosphate, D-glyceraldehyde 3-phosphate, glucose 6-phosphate/fructose 6-phosphate were down-regulated. These 
intermediates can be converted to glycogenic amino acid for the gluconeogenesis requirements [18,21]. The increases of glycolysis were also found in plasma and milk studies of HS dairy cows [4,5]. Second, the increased ketone in the liver of HS group, including acetoacetate and $\beta$-BHBA, suggested an enhancement of $\beta$-oxidation of free fatty acids to satisfy energy demands of the NEB body [20]. This kind of ketone metabolism was also detected in milk and plasma studies of HS dairy cows $[4,5]$. Third, marked changes of liver TCA metabolism in the HS group was an evidence of mitochondrial dysfunction and an energy crisis [19]. As far as we know, no publications had been reported about this TCA alterations in liver of HS dairy cows, and it may be a specific response to HS stimuli. Since TCA is a common metabolic pathway for various fuel molecules, such as amino acids, fatty acids, also the precursors of many biosysthesis, less perturbations of TCA responses to HS may be a gold reference for the selection of dairy cows more tolerable to HS conditions. Fourth, amino acid metabolism exhibited different alteration trends among liver, plasma, and milk due to different metabolic influence factors. In liver, amino acids play important role in gluconeogenesis, and most of the them expressed down-regulation. In plasma, catabolism of myoprotein exerted major influences on increased amino acids concentrations in HS dairy cows [4]. In milk, down-regulated amino acids were supposed to be the short supply from plasma into mammary glandular cells due to more distribution of plasma amino acids into gluconeogenesis or energy production rather than protein synthesis in mammary glandular cells [5]. Disturbed nucleotide metabolism was discovered in liver, not in plasma or milk, and this disturbance was supposed to be potential biomarkers in selection for tolerance to HS in dairy cattle [24,25]. Although nucleotide disturbance was not detected in HS plasma or milk several years ago of our previous studies, this alteration may be acquired in our next specific metabolic pathway or targeted metabolomics study due to our continuously improved detection sensitivity of analytical techniques and methods. Then, nucleotide can become practical biomarkers due to easily sample collection without slaughtering animals.

The metabolism of an animal is always in a state of dynamic equilibrium in which the influx of nutrients is balanced by the production of energy in catabolic and anabolic processes. Metabolic heat production of dairy cows increases with the level of milk synthesis, making high-yielding dairy cows extremely susceptible to environmental heat. When ambient temperatures exceed the thermoneutral zone of lactating dairy cows, feed intake declines, which contributes to reduction in milk production and loss of body weight [26]. As a result, dairy cows produce less metabolic heat and are less susceptible to environmental heat [26]. Besides the reduction in energy expenditure, metabolic adaptation to thermal stress also involves a shift in postabsorptive metabolism and nutrient partition- ing to reduce endogenous heat production.

Direct and indirect effects (e.g., reduced intake) of environment-induced hyperthermia must be distinguished because both heat-stressed and malnourished animals share common responses (i.e., reduced milk yield). A previous study reported that reduced nutrient intake accounted for just 35\% of HSinduced decreases in milk yield, with modest changes in the somatotropic axis possibly contributing to a portion of the remainder [27]. Thus, a combination of HS and reduced intake may have contributed to the results of the present study.

\section{CONCLUSION}

Global changes in metabolic networks suggest that biofluids (e.g., serum, plasma, and milk) may reflect changes in the physiological status of heat-stressed dairy cows. LC-MS techniques enable comprehensive analyses of metabolic profiles, and they can be used to identify potential biomarkers of HSinduced metabolic disorders in lactating dairy cows. Eight metabolites were identified as sensitive potential biomarkers in detecting the HS status of dairy cows. The data obtained from the metabolomics analyses of livers from dairy cows exposed to HS were highly consistent with potential biomarkers (e.g., glucose, lactate, pyruvate, acetoacetate, BHBA, fumaric acid, citric acid, choline, glycine, proline, isoleucine, leucine, urea, creatinine, and orotic acid) identified in analyses of candidate biomarkers in plasma or milk in heat-stressed lactating dairy cows. Additional analyses of other organs, such as the brain, mammary glands, hypothalamus, and pituitary tissues, are required to provide a more comprehensive perspective of the physiological mechanisms involved in HSinduced metabolic alterations.

\section{CONFLICT OF INTEREST}

We certify that there is no conflict of interest with any financial organization regarding the material discussed in the manuscript.

\section{ACKNOWLEDGMENTS}

This study was supported financially by National Key Research and Development Program of China (2016YFD0500503) and Introduction of International Advanced Agricultural Science and Technology Program (2016-X37). We thanks for the help of LipidALL technologies Ltd (China) in detecting all the samples.

\section{REFERENCES}

1. Wheelock JB, Rhoads RP, Vanbaale MJ, Sanders SR, Baumgard LH. Effects of heat stress on energetic metabolism in lactating 
Holstein cows. J Dairy Sci 2010;93:644-55.

2. Key N, Sneeringer S. Potential effects of climate change on the productivity of U.S. dairies. Am J Agric Econ 2014;1-21.

3. Baumgard LH, Wheelock JB, Sanders SR, et al. Postabsorptive carbohydrate adaptations to heat stress and monensin supplementation in lactating Holstein cows. J Dairy Sci 2011;94:562033.

4. Tian H, Wang W, Zheng N, et al. Identification of diagnostic biomarkers and metabolic pathway shifts of heat-stressed lactating dairy cows. J Proteomics 2015;125:17-28.

5. Tian $\mathrm{H}$, Zheng $\mathrm{N}$, Wang W, et al. Integrated metabolomics study of the milk of heat-stressed lactating dairy cows. Sci Rep 2016;6:24208.

6. Allen JD, Hall LW, Collier RJ, Smith JF. Effect of core body temperature, time of day, and climate conditions on behavioral patterns of lactating dairy cows experiencing mild to moderate heat stress. J Dairy Sci 2015;98:118-27.

7. Slimen B, Najar T, Ghram A, Abdrranna M. Heat stress effects on livestock: molecular, cellular and metabolic aspects, a review. J Anim Physiol Anim Nutr 2016;100:401-12.

8. Wang JP, Bu DP, Wang JQ, et al. Effect of saturated fatty acid supplementation on production and metabolism indices in heat-stressed mid-lactation dairy cows. J Dairy Sci 2010;93: 4121-7.

9. Cheng JB, Wang WY, Zheng N, et al. Natural period change of heat stress reveals unique "heat-stressed milk protein decrease syndrome" in mid-lactation dairy cows. China Anim Husb Vet Med 2014;41:73-84.

10.St-Pierre NR, Cobanov B, Schnitkey G. Economic losses from heat stress by US livestock industries. J Dairy Sci 2003;86:E52E77.

11. Bernabucci U, Biffani S, Buggiotti L, et al. The effects of heat stress in Italian Holstein dairy cattle. J Dairy Sci 2014;97:47186.

12. Fabris TF, Laporta J, Corra FN, et al. Effect of nutritional immunomodulation and heat stress during the dry period on subsequent performance of cows. J Dairy Sci 2017;100:673342.

13. Kirchgessner TG, Sleph P, Ostrowski J, et al. Beneficial and adverse eEffects of an LXR agonist on human lipid and lipoprotein metabolism and circulating neutrophils. Cell Metab 2016;24:223-33.

14. Puchalska P, Crawford PA. Multi-dimensional roles of ketone bodies in fuel metabolism, signaling, and therapeutics. Cell Metab 2017;25:262-84.
15. Siskos AP, Jain P, Römisch-Margl W, et al. Interlaboratory reproducibility of a targeted metabolomics platform for analysis of human serum and plasma. Anal Chem 2017;89:656-65.

16. de Castro NM, Yaqoob P, de la Fuente M, Baeza I, Claus SP. Premature impairment of methylation pathway and cardiac metabolic dysfunction in fa/fa obese Zucker rats. J Proteome Res 2013;12:1935-45.

17. Vander Heiden MG, Cantley LC, Thompson CB. Understanding the warburg effect: the metabolic requirements of cell proliferation. Science 2009;324:1029-33.

18. Gray LR, Sultana MR, Rauckhorst AJ, et al. Hepatic mitochondrial pyruvate carrier 1 is required for efficient regulation of gluconeogenesis and whole-body glucose homeostasis. Cell Metab 2015;22:669-81.

19. Ippolito DL, Lewis JA, Yu C, Leon LR, Stallings JD. Alteration in circulating metabolites during and after heat stress in the conscious rat: potential biomarkers of exposure and organspecific injury. BMC Physiol 2014;14:14.

20.Dunning KR, Russell DL, Robker RL. Lipids and oocyte developmental competence: the role of fatty acids and $\beta$-oxidation. Reproduction 2014;148:R15-R27.

21. Moreno KX, Moore CL, Burgess SC, et al. Production of hyperpolarized $13 \mathrm{CO} 2$ from [1-13C] pyruvate in perfused liver does reflect total anaplerosis but is not a reliable biomarker of glucose production. Metabolomics 2015;11:1144-56.

22. Monteiro AP, Guo JR, Weng XS, et al. Effect of maternal heat stress during the dry period on growth and metabolism of calves. J Dairy Sci 2016;99:3896-907.

23.Xu B, Chen M, Ji X, et al. Metabolomic profiles reveal key metabolic changes in heat stress-treated mouse Sertoli cells. Toxicol In Vitro 2015;29:1745-52.

24. Nguyen TT, Bowman PJ, Haile-Mariam M, Pryce JE, Hayes BJ. Genomic selection for tolerance to heat stress in Australian dairy cattle. J Dairy Sci 2016;99:2849-62.

25. Macciotta NPP, Biffani S, Bernabucci U, et al. Derivation and genome-wide association study of a principal componentbased measure of heat tolerance in dairy cattle. J Dairy Sci 2017;100:4683-97.

26. West JW. Effects of heat-stress on production in dairy cattle. J Dairy Sci 2003;86: 2131-44.

27. Rhoads ML, Rhoads RP, VanBaale MJ, et al. Effects of heat stress and plane of nutrition on lactating Holstein cows: I. Production, metabolism, and aspects of circulating somatotropin. J Dairy Sci 2009;92:1986-97. 